\title{
Multi-objective $H_{\infty}$ Control for Vehicle Active Suspension Systems with Random Actuator Delay
}

\author{
Hongyi Li, Honghai Liu, Steve Hand and Chris Hilton
}

\begin{abstract}
This paper is concerned with the problem of multi-objective $H_{\infty}$ control for vehicle active suspension systems with random actuator delay, which can be represented by signal probability distribution. First, the dynamical equations of a quarter-car suspension model are established for the control design purpose. Secondly, when taking into account vehicle performance requirements, namely, ride comfort, suspension deflection and the probability distributed actuator delay, we present the corresponding dynamic system, which will be transformed to the stochastic system for the problem of multi-objective $H_{\infty}$ controller design. Thirdly, based on the stochastic stability theory, the state feedback controller is proposed to render that the closed-loop system is exponentially stable in mean-square while simultaneously satisfying $H_{\infty}$ performance and the output constraint requirement. The presented condition is expressed in the form of convex optimization problems so that it can be efficiently solved via standard numerical software. Finally, a practical design example is given to demonstrate the effectiveness of the proposed method.
\end{abstract}

Keywords: Active suspension system; Actuator delay; Exponentially stability in mean-square; Multiobjective control; $H_{\infty}$ control.

This work was fully supported by a joint PhD scholarship of Portsmouth University and Protean Electric Ltd..

H. Li and H. Liu are with Intelligent Systems \& Biomedical Robotics Group, the School of Creative Technologies, University of Portsmouth, Portsmouth, PO1 2DJ, UK. Email: hongyi.li@port.ac.uk; honghai.liu@port.ac.uk

S. Hand is with the School of Creative Technologies, University of Portsmouth, Portsmouth, PO1 2DJ, UK. Email: steve.hand@port.ac.uk

C. Hilton is with Protean Electric Limited, Coxbridge Business Park, Surrey, GU10 5EH, UK. Email: chris.hilton@proteanelectric.com 


\section{INTRODUCTION}

Performance requirements of modern vehicle suspensions will include ride comfort, (i.e., it is to isolate passengers from vibration and shock arising from road roughness) good road holding, (i.e., it is to suppress the hop of the wheels so as to maintain firm and uninterrupted contact of wheels to road) and suspension deflection, (i.e., it is to keep suspension strokes within an allowable maximum [1]). these requirements are conflicted in principle which means that a compromise of the requirements must be achieved for better combination performances [2], [3].

Recently, considerable attention has been paid to improving the performances of vehicle suspensions in the literature [4]-[6]. It is evident that active suspension systems is an effective way to improve suspension performance [1], [7]. Active suspension control research is aimed at improving the ride performance which is generally quantified by sprung mass acceleration, and to maintain an acceptable level of suspension stroke and tyre deflection. To reach the target, various kinds of control techniques have been applied to active suspension control to improve the performance, such as fuzzy logic and neural network control [8], gain scheduling control [9], linear optimal control [10], adaptive control [11] and $H_{\infty}$ control [3], [12]. Among these methods, it has been proven that $H_{\infty}$ control methods [13] of vehicle active suspensions [3], [12] is a feasible way to manage the trade-off between conflicting performance i.e. means that minimization of suspension travel cannot be accomplished simultaneously with maximization of the ride comfort. Therefore, more recently, many researchers have paid growing attention to the $H_{\infty}$ control problem for active suspension vehicles and presented some results, for example, [3], [12], [14], [15].

It is evident that the time delay inherently existing in the vehicle suspension systems may be the source of poor performance and instability of the systems especially for the active control problem. Time delay or transportation lag is often encountered in many engineering systems [16], such as pneumatic and hydraulic systems, chemical processes, and long transmission lines. In general, the total delay in a control system is two fold. On one hand, time delay is caused by online data acquisition, filtering, calculating control forces, and transmitting the control force signals from a computer to the actuator. Specially, such time delay may be caused by the utilization of digital computers in control systems in that it takes finite time to carry out computation. However, the relevant computational delay can be modelled as constant and equal to the sampling period by using periodically driven sample and hold systems. Given today's computing power, such as digital-signal-processer-based system, this kind of delay will not play a substantial negative impact on the performance of a suspension control system since it can be estimated in 
advance. On the other hand, the delay known as floating time delay is taken by an actuator to build up the required control force. This kind of time delay usually depends on the particular dynamics of actuators [17], [18]. Due to the delay existing in actuators, the problem of controller design for active vehicle suspensions has been investigated to make the controller tolerate the time delay effect in [19], [20]. very recently, the authors in [3], [21] considered controller design for active suspensions with actuator delay.

Over the past years, there has been considerable research work reported to address the problems of $H_{\infty}$ control of active suspensions in the context of robustness and disturbance attenuation [2], [3], [12], [14], [15]. In [12], the authors presented the constrained control $H_{\infty}$ scheme for active suspensions with output and control constraint. The problem of $H_{\infty}$ controller design for a class of uncertain vehicle suspension systems with sampling measurements was addressed in [2]. In addition, the authors in [3], [22] proposed the $H_{\infty}$ controller design method for the vehicle suspension systems with actuator delay.

Motivated by the above observation, in this paper we focus on the problem of multi-objective $H_{\infty}$ control for active vehicle suspension systems with random actuator delay occurred according to its probability distribution. The random actuator delay is assumed to be time-varying and vary in a given range. First, we establish the dynamical equations of a quarter-car suspension model. Secondly, the vehicle performance requirements such as ride comfort, suspension deflection and the probability distributed actuator delay are considered for the presented dynamic system. Thirdly, based on the stochastic stability theory, a state feedback controller is proposed to ensure that the closed-loop system is exponentially stable in mean-square with $H_{\infty}$ performance and output constraint requirement simultaneously. The corresponding multi-objective $H_{\infty}$ controller existence condition can be expressed by the form of linear matrix inequalities (LMIs), which can be efficiently solved via standard numerical software [23]. Finally, a design example is employed to present the effectiveness of the proposed method. The remaining part of the paper is organized as follows: The problem to be addressed is presented in Section II and multiobjective $H_{\infty}$ controller design method is proposed in Section III, respectively. In Section IV, we present a design example to demonstrate the effectiveness of the developed approach and we conclude the paper in Section V.

Notation: The notation used in this paper is fairly standard. $\mathbb{R}^{n}$ denotes the $n$-dimensional Euclidean space, $\mathbb{R}^{n \times m}$ stands for the set of real $n \times m$ matrices. The superscript " $T$ " is denoted as matrix transposition; The notation $P>0(\geq 0)$ means that $P$ is real symmetric and positive definite (semidefinite). In symmetric block matrices or complex matrix expressions, we use an asterisk $(*)$ to represent a term that is induced by symmetry and $\operatorname{diag}\{\ldots\}$ stands for a block-diagonal matrix. The notation $\operatorname{sym}\{A\}$ is used to stand for $A+A^{T} . \mathcal{E}\{\cdot\}$ is used to denote the mathematical expectation. The space of 
square-integrable vector functions over $[0, \infty)$ is denoted by $L_{2}[0, \infty)$, and for $w=\{w(t)\} \in L_{2}[0, \infty)$, its norm is given by $\|w\|_{2}=\sqrt{\int_{t=0}^{\infty}|w(t)|^{2} \mathrm{~d} t}$. Matrices, if their dimensions are not explicitly stated, are assumed to be compatible for algebraic operations.

\section{PROBLEM Formulation}

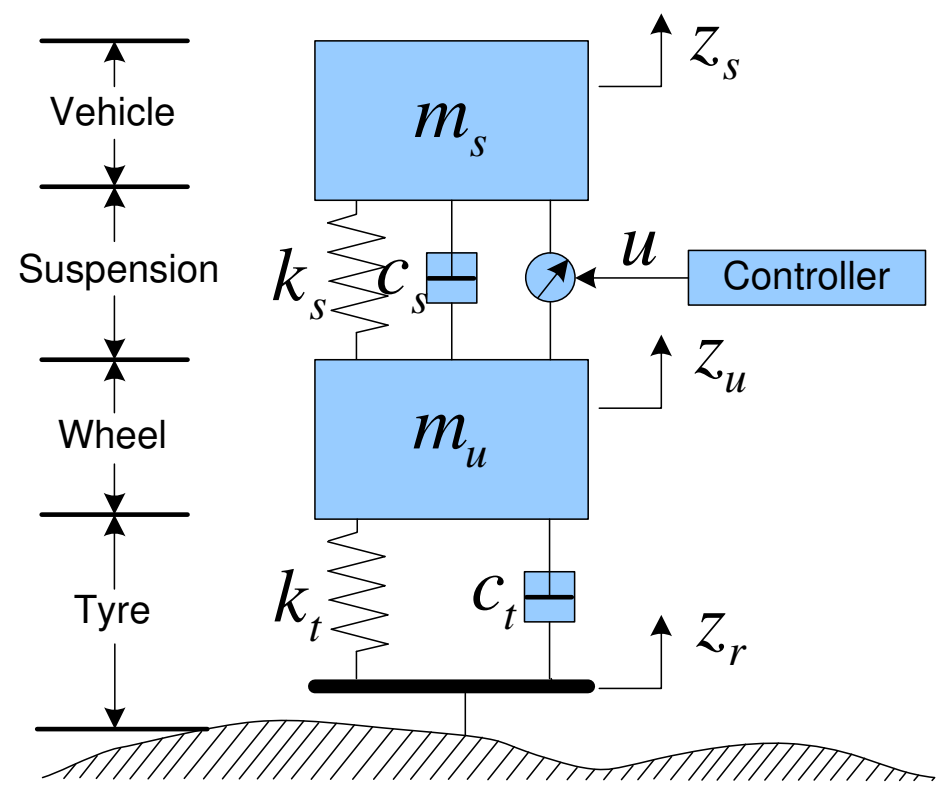

Fig. 1. A quarter-car model with an active suspension

The generalized quarter-car suspension model is shown in Fig. 1, in which $z_{s}$ and $z_{u}$ stands for the displacements of the sprung and unsprung masses, respectively; $z_{r}$ denotes the road displacement input; $u$ is the active input of the suspension system; $m_{s}$ is the sprung mass, which represents the car chassis; $m_{u}$ is the unsprung mass, which represents mass of the wheel assembly; $c_{s}$ and $k_{s}$ are damping and stiffness of the suspension system, respectively; $k_{t}$ and $c_{t}$ stand for compressibility and damping of the pneumatic tyre, respectively. Then, the equations of motion can be established as follows:

$$
\begin{aligned}
m_{s} \ddot{z}_{s}(t) & =c_{s}\left[\dot{z}_{u}(t)-\dot{z}_{s}(t)\right]+k_{s}\left[z_{u}(t)-z_{s}(t)\right]+u(t), \\
m_{u} \ddot{z}_{u}(t) & =c_{s}\left[\dot{z}_{s}(t)-\dot{z}_{u}(t)\right]+k_{s}\left[z_{s}(t)-z_{u}(t)\right]+k_{t}\left[z_{r}(t)-z_{u}(t)\right]+c_{t}\left[\dot{z}_{r}(t)-\dot{z}_{u}(t)\right]-u(t) .
\end{aligned}
$$


Let us define the following state variables:

\begin{tabular}{||l||l||}
\hline$x_{1}(t)=z_{s}(t)-z_{u}(t)$ & denotes the suspension deflection, \\
\hline$x_{2}(t)=z_{u}(t)-z_{r}(t)$ & denotes the tire deflection, \\
\hline$x_{3}(t)=\dot{z}_{s}(t)$ & denotes the sprung mass speed, \\
\hline$x_{4}(t)=\dot{z}_{u}(t)$ & denotes the unsprung mass speed. \\
\hline
\end{tabular}

Then we define the disturbance input $w(t)=\dot{z}_{r}(t)$ and the state vector as

$$
x(t)=\left[\begin{array}{llll}
x_{1}(t) & x_{2}(t) & x_{3}(t) & x_{4}(t)
\end{array}\right]^{T},
$$

next the dynamic equations in (1) can be expressed as the following state-space form:

$$
\dot{x}(t)=A x(t)+B_{1} w(t)+B u(t),
$$

where

$$
A=\left[\begin{array}{cccc}
0 & 0 & 1 & -1 \\
0 & 0 & 0 & 1 \\
-\frac{k_{s}}{m_{s}} & 0 & -\frac{c_{s}}{m_{s}} & \frac{c_{s}}{m_{s}} \\
\frac{k_{s}}{m_{u}} & -\frac{k_{t}}{m_{u}} & \frac{c_{s}}{m_{u}} & -\frac{c_{s}+c_{t}}{m_{u}}
\end{array}\right], \quad B=\left[\begin{array}{c}
0 \\
0 \\
\frac{1}{m_{s}} \\
-\frac{1}{m_{u}}
\end{array}\right], \quad B_{1}=\left[\begin{array}{c}
0 \\
-1 \\
0 \\
\frac{c_{t}}{m_{u}}
\end{array}\right]
$$

When dealing with the controller design for the active suspension system, the main performance requirement conditions, namely, ride comfort, and suspension deflection should be taken into account. Since ride comfort can be generally quantified by the body acceleration in the vertical direction, it is essential to choose body acceleration as the first control output, i.e., $\ddot{z}_{s}(t)$. so when we design the controller, one of our main objectives is to minimize the vertical acceleration $\ddot{z}_{s}(t)$. The $H_{\infty}$ norm is employed to measure the performance, whose value actually gives an upper bound of the root-meansquare gain. Hence, our goal is to minimize the $H_{\infty}$ norm of the transfer function from the disturbance $w(t)$ to the control output $z_{1}(t)=\ddot{z}_{s}(t)$ in order to improve ride comfort. Besides, due to the mechanical structure, the suspension stroke should not exceed the allowable maximum, that is,

$$
\left|z_{s}(t)-z_{u}(t)\right| \leq z_{\max }
$$

where $z_{\max }$ is the maximum suspension deflection. The controller should be able to ensure the car safety and prevent the suspension from hitting its travel limit in order to avoid ride comfort deterioration and mechanical structural damage.

Based on the above conditions, therefore, we select the $H_{\infty}$ norm to measure the body acceleration $\ddot{z}_{s}(t)$ being as a performance output, and the suspension stroke $z_{s}(t)-z_{u}(t)$ as a constrained output. 
Subsequently, the vehicle active suspension system can be described by the following state-space equations:

$$
\begin{aligned}
\dot{x}(t) & =A x(t)+B_{1} w(t)+B u(t), \\
z_{1}(t) & =C_{1} x(t)+D_{1} u(t), \\
z_{2}(t) & =C_{2} x(t),
\end{aligned}
$$

where $A, B_{1}$ and $B$ are defined in (2), and

$$
C_{1}=\left[\begin{array}{cccc}
-\frac{k_{s}}{m_{s}} & 0 & -\frac{c_{s}}{m_{s}} & \frac{c_{s}}{m_{s}}
\end{array}\right], \quad D_{1}=\frac{1}{m_{s}}, \quad C_{2}=\left[\begin{array}{cccc}
1 & 0 & 0 & 0
\end{array}\right] .
$$

For the vehicle active suspension system in (5), we suppose that the input $u(t)$ may or may not experience input delay, and may be dependent on probability distribution. We can describe the following two random events:

$$
\begin{cases}\text { Even 1: } & u(t) \text { does not experience input delay, } \\ \text { Even 2: } & u(t) \text { does experience input delay. }\end{cases}
$$

Let the stochastic variable $\delta(t)$ be defined as

$$
\delta(t)= \begin{cases}1, & \text { if Even } 1 \text { occurs } \\ 0, & \text { if Even } 2 \text { occurs. }\end{cases}
$$

As discussed in [24]-[27], $\delta(t)$ is a Markovian process and can be assumed to follow an exponential distribution of switchings, which satisfies,

$$
\left\{\begin{array}{l}
\operatorname{Prob}\{\delta(t)=1\}=\mathcal{E}\{\delta(t)\}=\bar{\delta}, \\
\operatorname{Prob}\{\delta(t)=0\}=1-\mathcal{E}\{\delta(t)\}=1-\bar{\delta},
\end{array}\right.
$$

where the constant $\bar{\delta} \in[0,1]$ shows the occurred probability of the event of no input delays. In what follows, we assume that the input is described by

$$
u(t)=\delta(t) K x(t)+(1-\delta(t)) K x(t-d(t)),
$$

where $K$ is a constant matrix to be determined later. $d(t)$ is the time-varying delay in the input channel which may be piecewise continuous satisfies the following condition.

There exist scalars $d_{1}, d_{2}$ and $\mu$ with $d_{2} \geq d_{1}>0$ such that

$$
0<d_{1} \leq d(t) \leq d_{2}, \quad \dot{d}(t) \leq \mu .
$$

It means that the time delay is a smooth function of $d(t)$ and its derivative is known to be the upper bounded by $\mu$. 
Under the controller in (8), the closed-loop system in (5) can be described as

$$
\begin{aligned}
\dot{x}(t) & =(A+\delta(t) B K) x(t)+(1-\delta(t)) B K x(t-d(t))+B_{1} w(t), \\
z_{1}(t) & =\left(C_{1}+\delta(t) D_{1} K\right) x(t)+(1-\delta(t)) D_{1} K x(t-d(t)), \\
z_{2}(t) & =C_{2} x(t) .
\end{aligned}
$$

We define a continuous initial function $x(t)=\phi(t), t \in\left[-2 d_{2}, 0\right]$ and we assume that $w(t)=0$ for $t \in\left[-2 d_{2}, 0\right]$. It is seen that system in (10) is a stochastic system with

$$
\mathcal{E}\{(\delta(t)-\bar{\delta})\}=0, \quad \mathcal{E}\left\{(\delta(t)-\bar{\delta})^{2}\right\}=\bar{\delta}(1-\bar{\delta})
$$

Subsequently, we rewrite the closed-loop systems in (10) as the following form:

$$
\begin{aligned}
\dot{x}(t)= & (A+\bar{\delta} B K) x(t)+(1-\bar{\delta}) B K x(t-d(t))+B_{1} w(t) \\
& +(\delta(t)-\bar{\delta})(B K x(t)-B K x(t-d(t))) \\
z_{1}(t)= & \left(C_{1}+\bar{\delta} D K\right) x(t)+(1-\bar{\delta}) D_{1} K x(t-d(t)) \\
& +(\delta(t)-\bar{\delta})\left(D_{1} K x(t)-D_{1} K x(t-d(t))\right) \\
z_{2}(t)= & C_{2} x(t) .
\end{aligned}
$$

For convenience, we have

$$
\begin{aligned}
f(t) & =(A+\bar{\delta} B K) x(t)+(1-\bar{\delta}) B K x(t-d(t))+B_{1} w(t) \\
g(t) & =B K x(t)-B K x(t-d(t))
\end{aligned}
$$

Since $f(t)$ and $g(t)$ in (11) satisfy the local Lipschitz condition and the linear growth condition, the existence and uniqueness of solution to (11) is guaranteed [28]. Moreover, under $w(t)=0$, it admits a trivial solution (equilibrium) $x \equiv 0$. The following definitions of exponential stability in mean square and $H_{\infty}$ performance requirements are given for the proof in the next section.

Definition 1: ( [27]) The system in (11) is said to be exponentially stable in mean square, if, under $u(t)=0$ and $w(t)=0$, there exist positive constant $\alpha$ and $\beta$, such that for all $t \geq 0$, the following inequality holds,

$$
\mathcal{E}\left\{\|x(\phi, t)\|^{2}\right\} \leq \alpha e^{-\beta t} \mathcal{E}\left\{\sup _{-2 d_{2} \leq s \leq 0}\|\phi(s)\|^{2}\right\} .
$$

Definition 2: Given a scalar $\gamma>0$, the system in (11) with $u(t)=0$ is said to be exponentially stable in mean square with disturbance attenuation $\gamma$ if it is exponentially stable in mean square and under zero 
initial conditions,

$$
\left\|z_{1}(t)\right\|_{E_{2}}<\gamma\|w(t)\|_{2}
$$

is satisfied for all nonzero $w(t) \in L_{2}[0, \infty)$, where

$$
\left\|z_{1}(t)\right\|_{E_{2}}=\left(\mathcal{E}\left\{\int_{0}^{\infty}\left|z_{1}(t)\right|^{2} \mathrm{~d} t\right\}\right)^{\frac{1}{2}} .
$$

Without loss of generality, it is assumed that $w \in L_{2}[0, \infty)$, and then we have $\|w\|_{2}^{2} \leq w_{\max }<\infty$. The objective of this paper is to determine a state controller in (8) such that

(1) the closed-loop system is exponentially stable in mean square;

(2) under zero initial condition, the closed-loop system guarantees that $\left\|z_{1}(t)\right\|_{E_{2}}<\gamma\|w(t)\|_{2}$ for all nonzero $w \in L_{2}[0, \infty)$, while the output constraint in (4) is satisfied

$$
\left|z_{2}(t)\right| \leq z_{2 \max }
$$

In the above proposed control strategy, the multiple requirements such as ride comfort, suspension deflection limit and random actuator delay are formulated in a unified framework, based on which the controller design is cast into a multiple-objective minimization problem.

\section{Multi-objective $H_{\infty}$ Performance Analysis}

In this section, we will first address the multi-objective $H_{\infty}$ performance analysis problem for the active suspension system in (11) in the following theorem.

Theorem 1: Consider the closed-loop system in (11). For given scalars $d_{1}, d_{2}, \mu, \bar{\delta}$ and a feedback gain $K$, if there exist matrices $P>0, Q_{1}>0, Q_{2}>0, Q_{3}>0, Z_{1}=\operatorname{diag}\left\{Z_{11}, Z_{11}\right\}>0$, $Z_{2}=\operatorname{diag}\left\{Z_{21}, Z_{21}\right\}>0, N_{1}, N_{2}$ and $N_{3}$ with appropriate dimensions such that the following LMIs hold:

$$
\begin{gathered}
{\left[\begin{array}{ccc}
\Omega+\Omega_{0} & \sqrt{d_{2}} N_{1}\left[I_{n}, I_{n}\right] & \sqrt{d_{21}} N_{2}\left[I_{n}, I_{n}\right] \\
* & -Z_{1} & 0 \\
* & * & -Z_{1}-Z_{2}
\end{array}\right]<0,} \\
{\left[\begin{array}{ccc}
\Omega+\Omega_{0} & \sqrt{d_{2}} N_{1}\left[I_{n}, I_{n}\right] & \sqrt{d_{21}} N_{3}\left[I_{n}, I_{n}\right] \\
* & -Z_{1} & 0 \\
* & * & -Z_{2}
\end{array}\right]<0,} \\
\\
{\left[\begin{array}{cc}
-I & \sqrt{\rho} C_{2} \\
* & -z_{\max }^{2} P
\end{array}\right]<0,}
\end{gathered}
$$


where

$$
\begin{aligned}
& \Omega=\operatorname{diag}\left\{Q_{1}+Q_{2}+Q_{3},(\mu-1) Q_{1},-Q_{2},-Q_{3},-\gamma^{2}\right\} \\
& +\operatorname{sym}\left\{W_{x}^{T} P P_{f}+N_{1} W_{N_{1}}+N_{2} W_{N_{2}}+N_{3} W_{N_{3}}\right\}, \\
& \Omega_{0}=\left[P_{f}^{T}, \delta_{0} P_{g}^{T}\right]\left[d_{2} Z_{1}+d_{21} Z_{2}\right]\left[P_{f}^{T}, \delta_{0} P_{g}^{T}\right]^{T}+Z_{11 w}^{T} Z_{11 w}+Z_{12 w}^{T} Z_{12 w},
\end{aligned}
$$

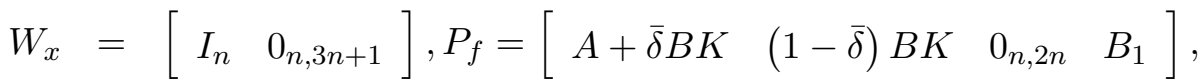

$$
\begin{aligned}
& P_{g}=\left[\begin{array}{lll}
B K & -B K & 0_{n, 2 n+1}
\end{array}\right], W_{N_{1}}=\left[\begin{array}{lll}
I_{n} & -I_{n} & 0_{n, 2 n+1}
\end{array}\right], \\
& W_{N_{2}}=\left[\begin{array}{lllll}
0_{n} & I_{n} & 0_{n} & -I_{n} & 0_{n, 1}
\end{array}\right], W_{N_{3}}=\left[\begin{array}{llll}
0_{n} & -I_{n} & I_{n} & 0_{n, n+1}
\end{array}\right] \text {, } \\
& Z_{11 w}=\left[\begin{array}{lll}
C_{1}+\bar{\delta} D_{1} K & (1-\bar{\delta}) D_{1} K & 0_{n, 2 n+1}
\end{array}\right], \\
& Z_{12 w}=\left[\begin{array}{lll}
\delta_{0} D_{1} K & -\delta_{0} D_{1} K & 0_{n, 2 n+1}
\end{array}\right], \quad d_{21}=d_{2}-d_{1}, \quad \delta_{0}=\sqrt{\bar{\delta}(1-\bar{\delta})}
\end{aligned}
$$

then a stabilizing controller in the form of (8) exists, such that

(1) the system is exponentially stable in mean square

(2) under zero initial condition, the closed-loop system guarantees that $\left\|z_{1}\right\|_{E_{2}}<\gamma\|w\|_{2}$ for all nonzero $w \in L_{2}[0, \infty)$;

(3) the control output constraint in (15) is guaranteed with the disturbance energy under the bound $w_{\max }=(\rho-V(0,0)) / \gamma^{2}$.

Proof. Firstly, we will show that the system in (10) with $w(t)=0$ is exponentially stable in mean square. Then, we will develop multi-objective $H_{\infty}$ performance analysis condition with the output constrain in (15). Let us consider the Lyapunov-Krasovskii functional as follows:

$$
\begin{aligned}
V\left(x_{t}, t\right)= & x^{T}(t) P x(t)+\int_{t-d(t)}^{t} x^{T}(s) Q_{1} x(s) \mathrm{d} s \\
& +\int_{t-d_{1}}^{t} x^{T}(s) Q_{2} x(s) \mathrm{d} s+\int_{t-d_{2}}^{t} x^{T}(s) Q_{3} x(s) \mathrm{d} s \\
& +\int_{-d_{2}}^{0} \int_{t+\theta}^{t}\left[\begin{array}{ll}
f^{T}(s) & \delta_{0} g^{T}(s)
\end{array}\right] Z_{1}\left[\begin{array}{ll}
f^{T}(s) & \delta_{0} g^{T}(s)
\end{array}\right]^{T} \mathrm{~d} s \mathrm{~d} \theta \\
& +\int_{-d_{2}}^{-d_{1}} \int_{t+\theta}^{t}\left[\begin{array}{lll}
f^{T}(s) & \delta_{0} g^{T}(s)
\end{array}\right] Z_{2}\left[\begin{array}{ll}
f^{T}(s) & \delta_{0} g^{T}(s)
\end{array}\right]^{T} \mathrm{~d} s \mathrm{~d} \theta,
\end{aligned}
$$

where $P>0, Q_{1}>0, Q_{2}>0, Q_{3}>0, Z_{1}>0$ and $Z_{2}>0$ are to be determined, $x_{t}=x(t+\sigma), \sigma \in$ $\left[-2 d_{2}, 0\right] . \mathcal{L} V\left(x_{t}, t\right)$ is used to denote the infinitesimal operator of $V\left(x_{t}, t\right)$, which is defined as

$$
\mathcal{L} V\left(x_{t}, t\right)=\lim _{\triangle \rightarrow 0+} \triangle^{-1}\left[\mathcal{E}\left\{V\left(x_{t+}, t+\triangle\right) \mid\left(x_{t}, t\right)\right\}-V\left(x_{t}, t\right)\right] .
$$


Then, it is can be seen from (19) and (20) that

$$
\begin{aligned}
& \mathcal{L} V\left(x_{t}, t\right)=2 x^{T}(t) \operatorname{Pf}(t)+x^{T}(t)\left(Q_{1}+Q_{2}+Q_{3}\right) x(t) \\
& -(1-d(t)) x^{T}(t-d(t)) Q_{1} x(t-d(t)) \\
& -x^{T}\left(t-d_{1}\right) Q_{2} x\left(t-d_{1}\right)-x^{T}\left(t-d_{2}\right) Q_{3} x\left(t-d_{2}\right) \\
& +\left[f^{T}(t), \delta_{0} g^{T}(t)\right]\left(d_{2} Z_{1}+d_{21} Z_{2}\right)\left[f^{T}(t), \delta_{0} g^{T}(t)\right]^{T} \\
& -\int_{t-d_{2}}^{t}\left[f^{T}(s), \delta_{0} g^{T}(s)\right] Z_{1}\left[f^{T}(s), \delta_{0} g^{T}(s)\right]^{T} \mathrm{~d} s \\
& -\int_{t-d_{2}}^{t-d_{1}}\left[f^{T}(s), \delta_{0} g^{T}(s)\right] Z_{2}\left[f^{T}(s), \delta_{0} g^{T}(s)\right]^{T} \mathrm{~d} s \\
& \leq 2 x^{T}(t) \operatorname{Pf}(t)+x^{T}(t)\left(Q_{1}+Q_{2}+Q_{3}\right) x(t) \\
& -(1-\mu) x^{T}(t-d(t)) Q_{1} x(t-d(t)) \\
& -x^{T}\left(t-d_{1}\right) Q_{2} x\left(t-d_{1}\right)-x^{T}\left(t-d_{2}\right) Q_{3} x\left(t-d_{2}\right) \\
& +\left[f^{T}(t), \delta_{0} g^{T}(t)\right]\left(d_{2} Z_{1}+d_{21} Z_{2}\right)\left[f^{T}(t), \delta_{0} g^{T}(t)\right]^{T} \\
& -\int_{t-d(t)}^{t}\left[f^{T}(s), \delta_{0} g^{T}(s)\right] Z_{1}\left[f^{T}(s), \delta_{0} g^{T}(s)\right]^{T} \mathrm{~d} s \\
& -\int_{t-d_{2}}^{t-d(t)}\left[f^{T}(s), \delta_{0} g^{T}(s)\right]\left(Z_{1}+Z_{2}\right)\left[f^{T}(s), \delta_{0} g^{T}(s)\right]^{T} \mathrm{~d} s \\
& -\int_{t-d(t)}^{t-d_{1}}\left[f^{T}(s), \delta_{0} g^{T}(s)\right] Z_{2}\left[f^{T}(s), \delta_{0} g^{T}(s)\right]^{T} \mathrm{~d} s .
\end{aligned}
$$

For any appropriately dimensioned matrices $N_{1}, N_{2}$ and $N_{3}$, it is easily to see that the following equalities hold:

$$
\begin{aligned}
2 \xi^{T}(t) N_{1}\left[x(t)-x(t-d(t))-\int_{t-d(t)}^{t} f(s) \mathrm{d} s-\int_{t-d(t)}^{t}(\delta(s)-\bar{\delta}) g(s) \mathrm{d} s\right] & =0 \\
2 \xi^{T}(t) N_{2}\left[x(t-d(t))-x\left(t-d_{2}\right)-\int_{t-d_{2}}^{t-d(t)} f(s) \mathrm{d} s-\int_{t-d_{2}}^{t-d(t)}(\delta(s)-\bar{\delta}) g(s) \mathrm{d} s\right] & =0, \\
2 \xi^{T}(t) N_{3}\left[x\left(t-d_{1}\right)-x(t-d(t))-\int_{t-d(t)}^{t-d_{1}} f(s) \mathrm{d} s-\int_{t-d(t)}^{t-d_{1}}(\delta(s)-\bar{\delta}) g(s) \mathrm{d} s\right] & =0,
\end{aligned}
$$

where

$$
\xi(t)=\left[\begin{array}{llll}
x^{T}(t) & x^{T}(t-d(t)) & x^{T}\left(t-d_{1}\right) & x^{T}\left(t-d_{2}\right)
\end{array}\right]^{T} .
$$


Furthermore, we have

$$
\begin{aligned}
& 2 \xi^{T}(t) N_{1}\left(-\int_{t-d(t)}^{t} f(s) \mathrm{d} s-\int_{t-d(t)}^{t}(\delta(s)-\bar{\delta}) g(s) \mathrm{d} s\right) \\
= & \int_{t-d(t)}^{t}\left\{-2 \xi^{T}(t) N_{1}\left[I_{n}, I_{n}\right]\left[f^{T}(s),(\delta(s)-\bar{\delta}) g^{T}(s)\right]^{T}\right\} \mathrm{d} s \\
\leq & \int_{t-d(t)}^{t} \xi^{T}(t) N_{1}\left[I_{n}, I_{n}\right] Z_{1}^{-1}\left[I_{n}, I_{n}\right]^{T} N_{1}^{T} \xi(t) \mathrm{d} s \\
& +\int_{t-d(t)}^{t}\left[f^{T}(s),(\delta(s)-\bar{\delta}) g^{T}(s)\right] Z_{1}\left[f^{T}(s),(\delta(s)-\bar{\delta}) g^{T}(s)\right]^{T} \mathrm{~d} s \\
= & d(t) \xi^{T}(t) N_{1}\left[I_{n}, I_{n}\right] Z_{1}^{-1}\left[I_{n}, I_{n}\right]^{T} N_{1}^{T} \xi(t) \\
& +\int_{t-d(t)}^{t}\left[f^{T}(s),(\delta(s)-\bar{\delta}) g^{T}(s)\right] Z_{1}\left[f^{T}(s),(\delta(s)-\bar{\delta}) g^{T}(s)\right]^{T} \mathrm{~d} s
\end{aligned}
$$

similarly,

$$
\begin{aligned}
& 2 \xi^{T}(t) N_{2}\left(-\int_{t-d_{2}}^{t-d(t)} f(s) \mathrm{d} s-\int_{t-d_{2}}^{t-d(t)}(\delta(s)-\bar{\delta}) g(s) \mathrm{d}\right) \\
\leq & \left(d_{2}-d(t)\right) N_{2} \xi^{T}(t)\left[I_{n}, I_{n}\right]\left(Z_{1}+Z_{2}\right)^{-1}\left[I_{n}, I_{n}\right]^{T} N_{2}^{T} \xi(t) \\
& +\int_{t-d_{2}}^{t-d(t)}\left[f^{T}(s),(\delta(s)-\bar{\delta}) g^{T}(s)\right]\left(Z_{1}+Z_{2}\right)\left[f^{T}(s),(\delta(s)-\bar{\delta}) g^{T}(s)\right]^{T} \mathrm{~d} s, \\
& 2 \xi^{T}(t) N_{3}\left(-\int_{t-d(t)}^{t-d_{1}} f(s) \mathrm{d} s-\int_{t-d(t)}^{t-d_{1}}(\delta(s)-\bar{\delta}) g(s) \mathrm{d} s\right) \\
\leq & \left(d(t)-d_{1}\right) \xi^{T}(t) N_{3}\left[I_{n}, I_{n}\right] Z_{2}^{-1}\left[I_{n}, I_{n}\right]^{T} N_{3}^{T} \xi(t) \\
& +\int_{t-d(t)}^{t-d_{1}}\left[f^{T}(s),(\delta(s)-\bar{\delta}) g^{T}(s)\right] Z_{2}\left[f^{T}(s),(\delta(s)-\bar{\delta}) g^{T}(s)\right]^{T} \mathrm{~d} s .
\end{aligned}
$$

To develop the stability condition, adding (22)-(24) to the right hand side of (21), using the inequalities (25)-(27), and taking the expectation on the both sides of (21), after some simple manipulation, we obtain,

$$
\begin{aligned}
\mathcal{E} \mathcal{L} V\left(x_{t}, t\right) \leq & \mathcal{E}\left\{\xi ^ { T } ( t ) \left[\hat{\Omega}+\hat{\Omega}_{0}+d_{2} N_{1}\left[I_{n}, I_{n}\right] Z_{1}^{-1}\left[I_{n}, I_{n}\right]^{T} N_{1}^{T}\right.\right. \\
& +\left(d_{2}-d(t)\right) N_{2}\left[I_{n}, I_{n}\right]\left(Z_{1}+Z_{2}\right)^{-1}\left[I_{n}, I_{n}\right]^{T} N_{2}^{T} \\
& \left.\left.+\left(d(t)-d_{1}\right) N_{3}\left[I_{n}, I_{n}\right] Z_{2}^{-1}\left[I_{n}, I_{n}\right]^{T} N_{3}^{T}\right] \xi(t)\right\} \\
= & \mathcal{E}\left\{\xi ^ { T } ( t ) \left[\frac { ( d _ { 2 } - d ( t ) ) } { d _ { 2 1 } } \left(\hat{\Omega}+\hat{\Omega}_{0}+d_{2} N_{1}\left[I_{n}, I_{n}\right] Z_{1}^{-1}\left[I_{n}, I_{n}\right]^{T} N_{1}^{T}\right.\right.\right. \\
& \left.+d_{21} N_{2}\left[I_{n}, I_{n}\right]\left(Z_{1}+Z_{2}\right)^{-1}\left[I_{n}, I_{n}\right]^{T} N_{2}^{T}\right) \\
& +\frac{\left(d(t)-d_{1}\right)}{d_{21}}\left(\hat{\Omega}+\hat{\Omega}_{0}+d_{2} N_{1}\left[I_{n}, I_{n}\right] Z_{1}^{-1}\left[I_{n}, I_{n}\right]^{T} N_{1}^{T}\right. \\
& \left.\left.\left.+d_{21} N_{3}\left[I_{n}, I_{n}\right] Z_{2}^{-1}\left[I_{n}, I_{n}\right]^{T} N_{3}^{T}\right)\right] \xi(t)\right\},
\end{aligned}
$$


where

$$
\begin{aligned}
& \hat{\Omega}=\operatorname{diag}\left\{Q_{1}+Q_{2}+Q_{3},(\mu-1) Q_{1},-Q_{2},-Q_{3}\right\} \\
& +\operatorname{sym}\left\{\hat{W}_{x}^{T} P \hat{P}_{f}+N_{1} \hat{W}_{N_{1}}+N_{2} \hat{W}_{N_{2}}+N_{3} \hat{W}_{N_{3}}\right\}, \\
& \hat{\Omega}_{0}=\left[\hat{P}_{f}^{T}, \delta_{0} \hat{P}_{g}^{T}\right]\left[d_{2} Z_{1}+d_{21} Z_{2}\right]\left[\hat{P}_{f}^{T}, \delta_{0} \hat{P}_{g}^{T}\right]^{T}, \\
& \hat{W}_{x}=\left[\begin{array}{ll}
I_{n} & 0_{n, 3 n}
\end{array}\right], \hat{P}_{f}=\left[\begin{array}{lll}
A+\bar{\delta} B K & (1-\bar{\delta}) B K & 0_{n, 2 n}
\end{array}\right], \\
& \hat{P}_{g}=\left[\begin{array}{lll}
B K & -B K & 0_{n, 2 n}
\end{array}\right], W_{\hat{N}_{1}}=\left[\begin{array}{lll}
I_{n} & -I_{n} & 0_{n, 2 n}
\end{array}\right], \\
& W_{\hat{N}_{2}}=\left[\begin{array}{llll}
0_{n} & I_{n} & 0_{n} & -I_{n}
\end{array}\right], W_{\hat{N}_{3}}=\left[\begin{array}{llll}
0_{n} & -I_{n} & I_{n} & 0_{n}
\end{array}\right] \text {. }
\end{aligned}
$$

Applying Schur complement to (16) and (17), we can conclude that

$$
\begin{aligned}
& \Xi_{1}=\hat{\Omega}+\hat{\Omega}_{0}+d_{2} N_{1}\left[I_{n}, I_{n}\right] Z_{1}^{-1}\left[I_{n}, I_{n}\right]^{T} N_{1}^{T}+d_{21} N_{2}\left[I_{n}, I_{n}\right]\left(Z_{1}+Z_{2}\right)^{-1}\left[I_{n}, I_{n}\right]^{T} N_{2}^{T}<0, \\
& \Xi_{2}=\hat{\Omega}+\hat{\Omega}_{0}+d_{2} N_{1}\left[I_{n}, I_{n}\right] Z_{1}^{-1}\left[I_{n}, I_{n}\right]^{T} N_{1}^{T}+d_{21} N_{3}\left[I_{n}, I_{n}\right] Z_{2}^{-1}\left[I_{n}, I_{n}\right]^{T} N_{3}^{T}<0 .
\end{aligned}
$$

Therefore, the following inequality holds,

$$
\mathcal{E} \mathcal{L} V\left(x_{t}, t\right) \leq-a\left(\|x(t)\|^{2}+\|x(t-d(t))\|^{2}\right),
$$

where

$$
a=\lambda_{\min }\left\{-\left(\frac{\left(d_{2}-d(t)\right)}{d_{21}} \Xi_{1}+\frac{\left(d(t)-d_{1}\right)}{d_{21}} \Xi_{2}\right)\right\}>0 .
$$

From the definitions of $V\left(x_{t}, t\right), f(t)$ and $g(t)$, there exist positive scalars $\eta_{1}, \eta_{2}$ and $\eta_{3}$ such that the following inequality holds

$$
V\left(x_{t}, t\right) \leq \eta_{1}\|x(t)\|^{2}+\eta_{2} \int_{t-d_{2}}^{t}\|x(s)\|^{2} \mathrm{~d} s+\eta_{3} \int_{t-d_{2}}^{t}\|x(s-d(s))\|^{2} \mathrm{~d} s .
$$

Choose $\beta>0$, such that

$$
\beta \max \left\{\eta_{1}+\eta_{2} d_{2} e^{\beta d_{2}}, \eta_{3} d_{2} e^{\beta d_{2}}\right\} \leq a .
$$

Therefore,

$$
\begin{aligned}
\mathcal{L}\left[e^{\beta t} V\left(x_{t}, t\right)\right] \leq & e^{\beta t}\left[\left(\beta \eta_{1}-a\right)\|x(t)\|^{2}-a\|x(t-d(t))\|^{2}\right. \\
& +\eta_{2} \int_{t-d_{2}}^{t}\|x(s)\|^{2} \mathrm{~d} s+\beta \eta_{3} \int_{t-d_{2}}^{t}\|x(s-d(s))\|^{2} \mathrm{~d} s
\end{aligned}
$$


By using Dynkin's formula, for $T>0$, we have

$$
\begin{aligned}
\mathcal{E}\left(e^{\beta T} V\left(x_{T}, T\right)\right) \leq & J_{1}+\left(\beta \eta_{1}-a\right) \mathcal{E}\left\{\int_{0}^{T} e^{\beta t}\|x(t)\|^{2} \mathrm{~d} t\right\} \\
& -a \mathcal{E}\left\{\int_{0}^{T} e^{\beta t}\|x(t-d(t))\|^{2} \mathrm{~d} t\right\} \\
& +\beta \eta_{2} \mathcal{E}\left\{\int_{0}^{T} \int_{t-d_{2}}^{t} e^{\beta t}\|x(s)\|^{2} \mathrm{~d} s \mathrm{~d} t\right\} \\
& +\beta \eta_{3} \mathcal{E}\left\{\int_{0}^{T} \int_{t-d_{2}}^{t} e^{\beta t}\|x(s-d(s))\|^{2} \mathrm{~d} s \mathrm{~d} t\right\}
\end{aligned}
$$

where

$$
J_{1}=\left[\eta_{1}+d_{2} \eta_{2}+d_{2} \eta_{3}\right] \sup _{-2 d_{2} \leq s \leq 0} \mathcal{E}\|\phi(s)\|^{2} .
$$

Consequently, by changing the integration sequence, the following inequalities hold,

$$
\begin{aligned}
\int_{0}^{T} \int_{t-d_{2}}^{t} e^{\beta t}\|x(s)\|^{2} \mathrm{~d} s \mathrm{~d} t & \leq \int_{-d_{2}}^{T}\left(\int_{s \vee 0}^{\left(s+d_{2}\right) \wedge T} e^{\beta t} \mathrm{~d} t\right)\|x(s)\|^{2} \mathrm{~d} s \\
& \leq \int_{-d_{2}}^{T} d_{2} e^{\beta\left(s+d_{2}\right)}\|x(t)\|^{2} \mathrm{~d} t \\
& \leq d_{2} e^{\beta d_{2}} \int_{0}^{T} e^{\beta t}\|x(t)\|^{2} \mathrm{~d} t+d_{2} e^{\beta d_{2}} \int_{-d_{2}}^{0}\|\phi(s)\|^{2} \mathrm{~d} s \\
& \leq d_{2} e^{\beta d_{2}} \int_{0}^{T} e^{\beta t}\|x(t)\|^{2} \mathrm{~d} t+d_{2}^{2} e^{\beta d_{2}} \sup _{-d_{2} \leq s \leq 0}\|\phi(s)\|^{2}, \\
& \leq d_{2} e^{\beta d_{2}} \int_{0}^{T} e^{\beta t}\|x(t)\|^{2} \mathrm{~d} t+d_{2}^{2} e^{\beta d_{2}} \sup _{-2 d_{2} \leq s \leq 0}\|\phi(s)\|^{2}, \\
\int_{0}^{T} \int_{t-d_{2}}^{t} e^{\beta t}\|x(s-d(s))\|^{2} \mathrm{~d} s \mathrm{~d} t & \leq d_{2} e^{\beta d_{2}} \int_{0}^{T} e^{\beta t}\|x(t-d(t))\|^{2} \mathrm{~d} t+d_{2}^{2} e^{\beta d_{2}} \sup _{-2 d_{2} \leq s \leq 0}\|\phi(s)\|^{2} .
\end{aligned}
$$

After substituting (34)-(35) into the right side of (32) and the using (30), we can obtain

$$
\mathcal{E}\left(e^{\beta T} V\left(x_{T}, T\right)\right) \leq J_{1}+J_{2},
$$

where

$$
J_{2}=\left(\beta \eta_{2} d_{2}^{2} e^{\beta d_{2}}+\beta \eta_{3} d_{2}^{2} e^{\beta d_{2}}\right) \sup _{-2 d_{2} \leq s \leq 0} \mathcal{E}\|\phi(s)\|^{2}
$$

So,

$$
\mathcal{E}\left\{\|x(\phi, T)\|^{2}\right\} \leq \frac{J_{1}+J_{2}}{\lambda_{\min }(P)} e^{-\beta T},
$$

then it can be shown that for any $T>0$,

$$
\mathcal{E}\left\{\|x(\phi, T)\|^{2}\right\} \leq \alpha e^{-\beta T} \sup _{-2 d_{2} \leq s \leq 0} \mathcal{E}\|\phi(s)\|^{2},
$$


where

$$
\alpha=\frac{1}{\lambda_{\min }(P)}\left[\eta_{1}+d_{2} \eta_{2}+d_{2} \eta_{3}+\beta \eta_{2} d_{2}^{2} e^{\beta d_{2}}+\beta \eta_{3} d_{2}^{2} e^{\beta d_{2}}\right] .
$$

Consequently, according to definition 1, the system in (11) is exponentially stable in mean square.

Furthermore, we will establish the $H_{\infty}$ performance analysis condition for the system in (11) under zero initial conditions. First, we define the Lyapunov-Krasovskii functional as in (19). For

$$
\bar{\xi}(t)=\left[\begin{array}{lllll}
x^{T}(t) & x^{T}(t-d(t)) & x^{T}\left(t-d_{1}\right) & x^{T}\left(t-d_{2}\right) & w^{T}(t)
\end{array}\right]^{T},
$$

by following the similar lines as in the above proof, one has

$$
\begin{aligned}
\mathcal{E} \mathcal{L} V\left(x_{t}, t\right) \leq & \mathcal{E}\left\{\overline { \xi } ^ { T } ( t ) \left[\check{\Omega}+\check{\Omega}_{0}+d_{2} N_{1}\left[I_{n}, I_{n}\right] Z_{1}^{-1}\left[I_{n}, I_{n}\right]^{T} N_{1}^{T}\right.\right. \\
& +\left(d_{2}-d(t)\right) N_{2}\left[I_{n}, I_{n}\right]\left(Z_{1}+Z_{2}\right)^{-1}\left[I_{n}, I_{n}\right]^{T} N_{2}^{T} \\
& \left.\left.+\left(d(t)-d_{1}\right) N_{3}\left[I_{n}, I_{n}\right] Z_{2}^{-1}\left[I_{n}, I_{n}\right]^{T} N_{3}^{T}\right] \bar{\xi}(t)\right\} \\
= & \mathcal{E}\left\{\overline { \xi } ^ { T } ( t ) \left[\frac { ( d _ { 2 } - d ( t ) ) } { d _ { 2 1 } } \left(\check{\Omega}+\check{\Omega}_{0}+d_{2} N_{1}\left[I_{n}, I_{n}\right] Z_{1}^{-1}\left[I_{n}, I_{n}\right]^{T} N_{1}^{T}\right.\right.\right. \\
& \left.+d_{21} N_{2}\left[I_{n}, I_{n}\right]\left(Z_{1}+Z_{2}\right)^{-1}\left[I_{n}, I_{n}\right]^{T} N_{2}^{T}\right) \\
& +\frac{\left(d(t)-d_{1}\right)}{d_{21}}\left(\check{\Omega}+\check{\Omega}_{0}+d_{2} N_{1}\left[I_{n}, I_{n}\right] Z_{1}^{-1}\left[I_{n}, I_{n}\right]^{T} N_{1}^{T}\right. \\
& \left.\left.\left.+d_{21} N_{3}\left[I_{n}, I_{n}\right] Z_{2}^{-1}\left[I_{n}, I_{n}\right]^{T} N_{3}^{T}\right)\right] \bar{\xi}(t)\right\},
\end{aligned}
$$

where

$$
\begin{aligned}
\check{\Omega}= & \operatorname{diag}\left\{Q_{1}+Q_{2}+Q_{3},(\mu-1) Q_{1},-Q_{2},-Q_{3}, 0\right\} \\
& +\operatorname{sym}\left\{W_{x}^{T} P P_{f}+N_{1} W_{N_{1}}+N_{2} W_{N_{2}}+N_{3} W_{N_{3}}\right\}, \\
\check{\Omega}_{0}= & {\left[P_{f}^{T}, \delta_{0} P_{g}^{T}\right]\left[d_{2} Z_{1}+d_{21} Z_{2}\right]\left[P_{f}^{T}, \delta_{0} P_{g}^{T}\right]^{T} . }
\end{aligned}
$$

Thus, we can show that

$$
\begin{aligned}
& \mathcal{E}\left\{z_{1}^{T}(t) z_{1}(t)-\gamma^{2} w^{T}(t) w(t)+\mathcal{L} V\left(x_{t}, t\right)\right\} \\
\leq & \mathcal{E}\left\{\overline { \xi } ^ { T } ( t ) \left[\frac { ( d _ { 2 } - d ( t ) ) } { d _ { 2 1 } } \left(\Omega+\Omega_{0}+d_{2} N_{1}\left[I_{n}, I_{n}\right] Z_{1}^{-1}\left[I_{n}, I_{n}\right]^{T} N_{1}^{T}\right.\right.\right. \\
& \left.+d_{21} N_{2}\left[I_{n}, I_{n}\right]\left(Z_{1}+Z_{2}\right)^{-1}\left[I_{n}, I_{n}\right]^{T} N_{2}^{T}\right) \\
& +\frac{\left(d(t)-d_{1}\right)}{d_{21}}\left(\Omega+\Omega_{0}+d_{2} N_{1}\left[I_{n}, I_{n}\right] Z_{1}^{-1}\left[I_{n}, I_{n}\right]^{T} N_{1}^{T}\right. \\
& \left.\left.\left.+d_{21} N_{3}\left[I_{n}, I_{n}\right] Z_{2}^{-1}\left[I_{n}, I_{n}\right]^{T} N_{3}^{T}\right)\right] \bar{\xi}(t)\right\} .
\end{aligned}
$$


By using Schur complement to (16)-(17), we have

$$
\mathcal{E}\left\{z_{1}^{T}(t) z_{1}(t)-\gamma^{2} w^{T}(t) w(t)+\mathcal{L} V\left(x_{t}, t\right)\right\}<0,
$$

for all nonzero $w \in L_{2}[0, \infty)$. Because $V(\phi(t), 0)=0$ under zero initial condition, that is, $\phi(t)=0$ for $t \in\left[-2 d_{2}, 0\right]$. Thus, by Itô's formula, after integrating both sides of (37), we obtain $\left\|z_{1}(t)\right\|_{E_{2}}<$ $\gamma\|w(t)\|_{2}$ for all nonzero $w \in L_{2}[0, \infty)$, and the $H_{\infty}$ performance is established.

In the following section, we consider the problems of the output constraints. From (37), it can be seen that

$$
\mathcal{E} \mathcal{L} V\left(x_{t}, t\right)-\gamma^{2} w^{T}(t) w(t)<0
$$

After integrating both sides of the above inequality from zero to any $t>0$, we obtain

$$
\mathcal{E} V\left(x_{t}, t\right)-V(0,0)<\gamma^{2} \int_{0}^{t} w^{T}(\tau) w(\tau) \mathrm{d} \tau<\gamma^{2}\|w\|_{2}^{2}
$$

From the definition of the Lyapunov functional in (19), we obtain $x^{T}(t) P x(t)<\rho$, with $\rho=\gamma^{2} w_{\max }+$ $V(0,0)$. Consider

$$
\begin{aligned}
\max _{t>0}\left|z_{2}(t)\right|^{2} & =\max _{t>0}\left\|x^{T}(t) C_{2}^{T} C_{2} x(t)\right\|_{2} \\
& =\max _{t>0}\left\|x^{T}(t) P^{\frac{1}{2}} P^{-\frac{1}{2}} C_{2}^{T} C_{2} P^{-\frac{1}{2}} P^{\frac{1}{2}} x(t)\right\|_{2} \\
& <\rho \cdot \theta_{\max }\left(P^{-\frac{1}{2}} C_{2}^{T} C_{2} P^{-\frac{1}{2}}\right),
\end{aligned}
$$

where $\theta_{\max }(\cdot)$ represents maximal eigenvalue. From the above inequality, we know that the constraint (15) is guaranteed, if

$$
\rho \cdot P^{-\frac{1}{2}} C_{2}^{T} C_{2} P^{-\frac{1}{2}}<z_{\max }^{2} I
$$

By Schur complement, (18) are equivalent to (38). We complete the proof.

\section{Multi-objective $H_{\infty}$ Controller Design}

Based on the $H_{\infty}$ performance analysis condition proposed in Theorem 1, the $H_{\infty}$ controller existence condition for the active suspension system in (10) is developed in the following theorem.

Theorem 2: Consider the closed-loop system in (11). For given constants $d_{1}, d_{2}, \mu$ and $\bar{\delta}$, if there exist matrices $Y, X>0, \bar{Q}_{1}>0, \bar{Q}_{2}>0, \bar{Q}_{3}>0, \bar{Z}_{1}=\operatorname{diag}\left\{\bar{Z}_{11}, \bar{Z}_{11}\right\}>0, \bar{Z}_{2}=\operatorname{diag}\left\{\bar{Z}_{21}, \bar{Z}_{21}\right\}>0$, 
$\bar{N}_{1}, \bar{N}_{2}$, and $\bar{N}_{3}$ with appropriate dimensions such that the following LMIs hold:

$$
\begin{gathered}
{\left[\begin{array}{cccc}
\bar{\Omega} & \sqrt{d_{2}} \bar{N}_{1}\left[I_{n}, I_{n}\right] & \sqrt{d_{21}} \bar{N}_{2}\left[I_{n}, I_{n}\right] & \Upsilon_{1} \\
* & \bar{Z}_{1}-2 \hat{X} & 0 & 0 \\
* & * & \bar{Z}_{1}+\bar{Z}_{2}-4 \hat{X} & 0 \\
* & * & * & \Upsilon_{2}
\end{array}\right]<0,} \\
{\left[\begin{array}{cccc}
\bar{\Omega} & \sqrt{d_{2}} \bar{N}_{1}\left[I_{n}, I_{n}\right] & \sqrt{d_{21}} \bar{N}_{3}\left[I_{n}, I_{n}\right] & \Upsilon_{1} \\
* & \bar{Z}_{1}-2 \hat{X} & 0 & 0 \\
* & \bar{Z}_{2}-2 \hat{X} & 0 \\
* & & * & \Upsilon_{2}
\end{array}\right]<0,} \\
{\left[\begin{array}{cc}
* & \sqrt{\rho} C_{2} X \\
* & -z_{\max }^{2} X
\end{array}\right]<0,}
\end{gathered}
$$

where

$$
\begin{aligned}
& \bar{\Omega}=\operatorname{diag}\left\{\bar{Q}_{1}+\bar{Q}_{2}+\bar{Q}_{3},(\mu-1) \bar{Q}_{1},-\bar{Q}_{2},-\bar{Q}_{3},-\gamma^{2}\right\} \\
& +\operatorname{sym}\left\{W_{x}^{T} \bar{P}_{f}+\bar{N}_{1} W_{N_{1}}+\bar{N}_{2} W_{N_{2}}+\bar{N}_{3} W_{N_{3}}\right\},
\end{aligned}
$$

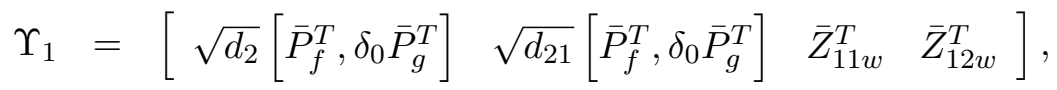

$$
\begin{aligned}
& \Upsilon_{2}=\operatorname{diag}\left\{-\bar{Z}_{1},-\bar{Z}_{2},-1,-1\right\}
\end{aligned}
$$

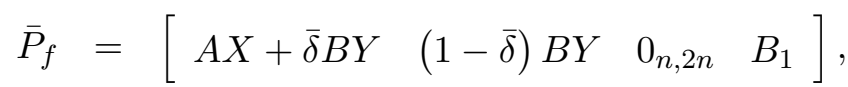

$$
\begin{aligned}
& \bar{P}_{g}=\left[\begin{array}{lll}
B Y & -B Y & 0_{n, 2 n+1}
\end{array}\right], W_{N_{1}}=\left[\begin{array}{lll}
I_{n} & -I_{n} & 0_{n, 2 n+1}
\end{array}\right], \\
& W_{N_{2}}=\left[\begin{array}{lllll}
0_{n} & I_{n} & 0_{n} & -I_{n} & 0_{n, 1}
\end{array}\right], W_{N_{3}}=\left[\begin{array}{llll}
0_{n} & -I_{n} & I_{n} & 0_{n, n+1}
\end{array}\right], \\
& \bar{Z}_{11 w}=\left[\begin{array}{lll}
C_{1} X+\bar{\delta} D_{1} Y & (1-\bar{\delta}) D_{1} Y & 0_{n, 2 n+1}
\end{array}\right], \\
& \bar{Z}_{12 w}=\left[\begin{array}{lll}
\delta_{0} D_{1} Y & -\delta_{0} D_{1} Y & 0_{n, 2 n+1}
\end{array}\right], \hat{X}=\operatorname{diag}\{X, X\},
\end{aligned}
$$

then a stabilizing controller in the form of (8) exists, such that

(1) the closed-loop system is exponentially stable in mean square;

(2) under zero initial condition, the closed-loop system guarantees that $\left\|z_{1}\right\|_{E_{2}}<\gamma\|w\|_{2}$ for all nonzero $w \in L_{2}[0, \infty)$;

(3) the control output constraint in (15) is guaranteed with the disturbance energy under the bound $w_{\max }=(\rho-V(0,0)) / \gamma^{2}$. 
Moreover, if inequalities (39)-(41) have a feasible solution, then the control gain $K$ in (8) is given by $K=Y X^{-1}$.

Proof. Due to the relationship

$$
\left(\bar{Z}_{e}-\hat{X}\right) \bar{Z}_{e}^{-1}\left(\bar{Z}_{e}-\hat{X}\right) \geq 0, \quad e=1,2
$$

we know

$$
-\hat{X} \bar{Z}_{e}^{-1} \hat{X} \leq \bar{Z}_{e}-2 \hat{X}, \quad e=1,2
$$

From (39)-(40), we have

$$
\begin{aligned}
& {\left[\begin{array}{cccc}
\bar{\Omega} & \sqrt{d_{2}} \bar{N}_{1}\left[I_{n}, I_{n}\right] & \sqrt{d_{21}} \bar{N}_{2}\left[I_{n}, I_{n}\right] & \Upsilon_{1} \\
* & -\hat{X} \bar{Z}_{1}^{-1} \hat{X}_{1} & 0 & 0 \\
* & * & -\hat{X}\left(\bar{Z}_{1}^{-1}+\bar{Z}_{2}^{-1}\right) \hat{X}_{1} & 0 \\
* & * & * & \Upsilon_{2}
\end{array}\right]<0,} \\
& {\left[\begin{array}{cccc}
\bar{\Omega} & \sqrt{d_{2}} \bar{N}_{1}\left[I_{n}, I_{n}\right] & \sqrt{d_{21}} \bar{N}_{3}\left[I_{n}, I_{n}\right] & \Upsilon_{1} \\
* & -\hat{X} \bar{Z}_{1}^{-1} \hat{X}_{1} & 0 & 0 \\
* & * & -\hat{X} \bar{Z}_{2}^{-1} \hat{X}_{1} & 0 \\
* & * & * & \Upsilon_{2}
\end{array}\right]<0 .}
\end{aligned}
$$

Now, introduce the following matrices

$$
\Theta=\operatorname{diag}\left\{\Theta_{1}, \Theta_{2}, \Theta_{3}, \Theta_{4}, 1,1\right\}
$$

where

$$
\begin{aligned}
& \Theta_{1}=\operatorname{diag}\left\{X^{-1}, X^{-1}, X^{-1}, X^{-1}, 1\right\}, \Theta_{2}=\operatorname{diag}\left\{\hat{X}^{-1}, \hat{X}^{-1}\right\}, \\
& \Theta_{3}=\operatorname{diag}\left\{I_{n}, I_{n}\right\}, \Theta_{4}=\operatorname{diag}\left\{I_{n}, I_{n}\right\}, \Theta_{5}=\operatorname{diag}\left\{X^{-1}, X^{-1}, X^{-1}\right\} .
\end{aligned}
$$

After setting

$$
\begin{aligned}
P & =X^{-1}, \quad Z_{e}=\bar{Z}_{e}^{-1}>0, \quad(e=1,2, h=1,2,3) \\
Q_{h} & =X^{-1} \bar{Q}_{h} X^{-1}, \quad\left[\begin{array}{lll}
N_{1} & N_{2} & N_{3}
\end{array}\right]=\Theta_{1}\left[\begin{array}{lll}
\bar{N}_{1} & \bar{N}_{2} & \bar{N}_{3}
\end{array}\right] \Theta_{5} .
\end{aligned}
$$

Pre- and post multiplying (43) and (44) by $\Theta^{T}$ and $\Theta$ respectively, we know that conditions in (16) and (17) hold by using Schur complement.

On the other hand, (41) is equivalent to (18) by performing a simple congruence transformation with $\operatorname{diag}\left\{I, X^{-1}\right\}$. Therefore, all the conditions in Theorem 1 are satisfied. The proof is completed. 
Remark 1: The random input delay idea used in this paper is motivated by the random sensor delay and random input delay proposed by [24]-[27]. In detail, the authors in [24] presented a controller design strategy for discrete-time networked systems with random communication delays. In [25], [26], the stability and stabilization problems for networked control systems and Takagi-Sugeno fuzzy systems with stochastic input delays were investigated respectively. The authors in [27] designed observer-based output feedback controller for continuous-time networked systems with random sensor delay, while the stability and stabilization conditions for the systems with random input delay have been developed. There exist few results on multi-objective $H_{\infty}$ control for the systems with random input delay. Based on the observation, the multi-objective $H_{\infty}$ control problem has been investigated for a quarter-car active suspension system with random input delay in this paper. Simulation results will be given in the following section to illustrate the effectiveness of the proposed control strategy.

Remark 2: It is can be seen from Theorem 2 that the condition is presented in the framework of LMIs both over the matrix variables and the objective scalar $\gamma$, which implies that $\gamma$ can be included as an optimization variable to obtain a lower bound of the guaranteed $H_{\infty}$ performance. Based on the condition in Theorem 2, the robust $H_{\infty}$ controller can be obtained with the minimal $\gamma$ by solving the following convex optimization problem:

$$
\begin{gathered}
\min \gamma \quad \text { s.t. } \quad(39)-(41) \\
X>0, \bar{Q}_{1}>0, \bar{Q}_{2}>0, \bar{Q}_{3}>0, \bar{Z}_{1}>0, \bar{Z}_{2}>0, \bar{K}, \bar{N}_{1}, \bar{N}_{2}, \bar{N}_{2} .
\end{gathered}
$$

\section{A Design Example}

In this section, a design example is provided to demonstrate the effectiveness of the proposed method. A quarter-car model parameters borrowed from [3] is listed in Table I for the following controller design.

TABLE I

QUARTER-CAR MODEL PARAMETERS

\begin{tabular}{||c||c||c||c||c||c||}
\hline$m_{s}$ & $m_{u}$ & $k_{s}$ & $k_{t}$ & $c_{s}$ & $c_{t}$ \\
\hline $973 \mathrm{~kg}$ & $114 \mathrm{~kg}$ & $42720 \mathrm{~N} / \mathrm{m}$ & $101115 \mathrm{~N} / \mathrm{m}$ & $1095 \mathrm{Ns} / \mathrm{m}$ & $14.6 \mathrm{Ns} / \mathrm{m}$ \\
\hline
\end{tabular}

The objective of this paper is to design the desired controller to ensure that the sprung mass acceleration $z_{1}(t)$ is as small as possible and the suspension deflection is below the maximum allowable suspension 
stroke $z_{\max }=0.08 \mathrm{~m}$ in (15). It is assumed that the delay $d(t)=7.5+2.5 \sin (t / 25) \mathrm{ms}$ satisfies $d_{1}=5$ $\mathrm{ms}, d_{2}=10 \mathrm{~ms}$ and $\mu=0.1$. Here, we choose $\rho=1$ as discussed in [12]. Firstly, for $\bar{\delta}=0$, by solving the convex optimization problem formulated in (45), it is found that the minimum guaranteed closed-loop $H_{\infty}$ performance obtained is $\gamma_{\min }=5.9996$, and admissible control gain matrix is given

$$
K=10^{4} \times\left[\begin{array}{llll}
-2.8881 & 0.2487 & -1.8410 & 0.1826
\end{array}\right] .
$$

Moreover, to present more detailed results on $H_{\infty}$ controller design for different $\bar{\delta}$, Table II lists the admissible controller gain matrices and the corresponding minimum guaranteed closed-loop $H_{\infty}$ performance indexes.

TABLE II

$H_{\infty}$ PERFORMANCE INDEX $\gamma_{\min }$ AND $K$ FOR DIFFERENT $\bar{\delta}$

\begin{tabular}{|c|c|c|c|c|c|c|}
\hline $\bar{\delta}$ & $\gamma_{\min }$ & & $K$ & & & \\
\hline 0.1 & 5.8982 & $K=10^{4} \times$ & -4.6681 & 1.1413 & -2.3007 & 0.1979 \\
\hline 0.2 & 5.8226 & $K=10^{4} \times$ & -4.3687 & 2.4833 & -2.3089 & 0.2760 \\
\hline 0.3 & 5.7563 & $K=10^{4} \times$ & -5.8424 & 1.7648 & -2.6021 & 0.2063 \\
\hline 0.4 & 5.7067 & $K=10^{4} \times$ & -7.1058 & 2.5891 & -2.9293 & 0.2187 \\
\hline 0.5 & 5.6527 & $K=10^{4} \times$ & -9.3258 & 3.5727 & -3.4869 & 0.2214 \\
\hline 0.6 & 5.6149 & $K=10^{5} \times$ & -1.1894 & 0.4943 & -0.4138 & 0.0229 \\
\hline 0.7 & 5.5940 & $K=10^{5} \times$ & -1.3334 & 0.7146 & -0.4561 & 0.0283 \\
\hline 0.8 & 5.5728 & $K=10^{5} \times$ & -1.3770 & 1.0997 & -0.4844 & 0.0426 \\
\hline 0.9 & 5.5562 & $K=10^{5} \times$ & -0.8263 & 1.9170 & -0.3995 & 0.0875 \\
\hline 1 & 5.5517 & $K=10^{5} \times$ & -0.0543 & 1.5399 & -0.2004 & 0.0844 \\
\hline
\end{tabular}

To further show the effectiveness of the proposed method, we consider the constant delay, that is $d_{2}=d_{1}$ and $\mu=0$ for $\bar{\delta}=0, \bar{\delta}=0.5$ and $\bar{\delta}=1$. By solving the convex optimization problem formulated in (45), the minimum guaranteed closed-loop $H_{\infty}$ performance index and admissible controller gain matrices are listed in Tables III-V for $\bar{\delta}=0, \bar{\delta}=0.5$ and $\bar{\delta}=1$ respectively. From Table II, we can see that the closed-loop system is exponentially stable in mean square and synchronously satisfies the guaranteed $H_{\infty}$ performance $\gamma_{\text {min }}$ which is decreasing with $\bar{\delta}$ increasing. In addition, it can be seen from Tables III-V that the minimum guaranteed $H_{\infty}$ performance $\gamma_{\min }$ decreases when $\bar{\delta}$ enlarges for the same constant delay, while under the same $\bar{\delta}$, the closed-loop performance $\gamma_{\min }$ is decreased with the constant delay increasing. 
TABLE III

$H_{\infty}$ PERFORMANCE INDEX $\gamma_{\text {min }}$ AND $K$ FOR DIFFERENT $\bar{\delta}=0$

\begin{tabular}{||c||c||ccc||}
\hline$d_{2}=d_{1}$ & $\gamma_{\min }$ & $K$ & & \\
\hline $5 \mathrm{~ms}$ & 5.4376 & $K=10^{5} \times\left[\begin{array}{llll||}-3.1526 & 0.3409 & -0.8660 & -0.0056\end{array}\right]$ \\
$10 \mathrm{~ms}$ & 5.7417 & $K=10^{4} \times\left[\begin{array}{llll}-5.5911 & 0.2482 & -2.4751 & 0.1443\end{array}\right]$ \\
\hline
\end{tabular}

TABLE IV

$H_{\infty}$ PERFORMANCE INDEX $\gamma_{\min }$ AND $K$ FOR DIFFERENT $\bar{\delta}=0.5$

\begin{tabular}{||c||c||ccc||}
\hline$d_{2}=d_{1}$ & $\gamma_{\min }$ & $K$ & & \\
\hline $5 \mathrm{~ms}$ & 5.4156 & $K=10^{5} \times\left[\begin{array}{llll}-7.0378 & 0.3057 & -1.7584 & -0.0652\end{array}\right]$ \\
$10 \mathrm{~ms}$ & 5.5339 & $K=10^{5} \times\left[\begin{array}{llll}-1.6413 & 0.3250 & -0.5115 & 0.0104\end{array}\right]$ \\
\hline
\end{tabular}

In order to evaluate the suspension characteristics with respect to ride comfort and working space of the suspension, the variability of the road profiles is taken into account. In the context of active suspension performance, road disturbances can be generally assumed as shocks. Shocks are discrete events of relatively short duration and high intensity, caused by, for example, a pronounced bump or pothole on an otherwise smooth road surface. In this work, this case of road profile is considered first to reveal the transient response characteristic, which is given by

$$
z_{r}(t)= \begin{cases}\frac{A}{2}\left(1-\cos \left(\frac{2 \pi V}{L} t\right)\right), & \text { if } 0 \leq t \leq \frac{L}{V} \\ 0, & \text { if } t>\frac{L}{V}\end{cases}
$$

where $A$ and $L$ are the height and the length of the bump. Assume $A=60 \mathrm{~mm}, L=5 \mathrm{~m}$ and the vehicle forward velocity as $V=35(\mathrm{~km} / \mathrm{h})$.

TABLE V

$H_{\infty}$ PERFORMANCE INDEX $\gamma_{\min }$ AND $K$ FOR DIFFERENT $\bar{\delta}=1$

\begin{tabular}{||c||c||ccc||}
\hline$d_{2}=d_{1}$ & $\gamma_{\min }$ & $K$ & & \\
\hline $5 \mathrm{~ms}$ & 5.4038 & $K=10^{6} \times\left[\begin{array}{llll}-4.1738 & -0.2627 & -0.9622 & -0.0685\end{array}\right]$ \\
$10 \mathrm{~ms}$ & 5.4641 & $K=10^{6} \times\left[\begin{array}{llll}-1.0251 & 0.2554 & -0.2610 & -0.0045\end{array}\right]$ \\
\hline
\end{tabular}


In this paper, the bump responses of the open-loop $(u(t)=0$, passive mode) and closed-loop (active mode) system with the controller $K$ listed in Table II for $\bar{\delta}=0, \bar{\delta}=0.5$ and $\bar{\delta}=1$ are showed Fig. 2. These figures plot the body vertical accelerations, suspension strokes, tyre deflections and active force for the open-loop and closed-loop system, respectively. They demonstrate that the closed-loop system is exponentially stable in mean square and has a better performance than the open-loop system, while the closed-loop system can guarantee the required performances such as the sprung mass acceleration $z_{1}(t)$ is as small as possible, the suspension deflection is below the maximum allowable suspension stroke $z_{\max }=0.08 \mathrm{~m}$. In addition, we can see from Fig. 2 that the active control forces of the closedloop systems, which are confined within a reasonable range which can be generated by hydraulic or electrorheological actuators in practice.
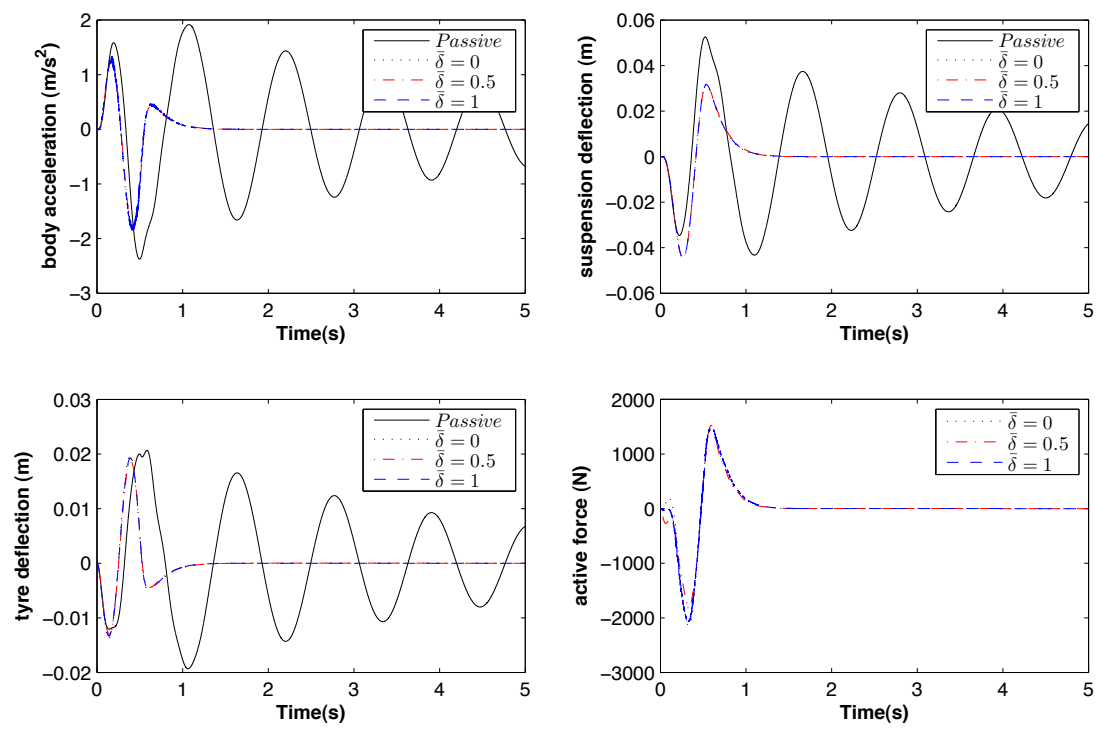

Fig. 2. Vertical accelerations, suspension strokes, tyre deflection and active force for system

Finally, by using the control gain matrices obtained in Table III-V, the responses of body vertical accelerations, suspension strokes, tyre deflection and active force for the closed-loop systems under different constant delays are illustrated in Fig. 3-5, from which it can be seen that the closed-loop system performance can be guaranteed. From the above figures, we know that the effectiveness of the proposed controller design method is confirmed. 

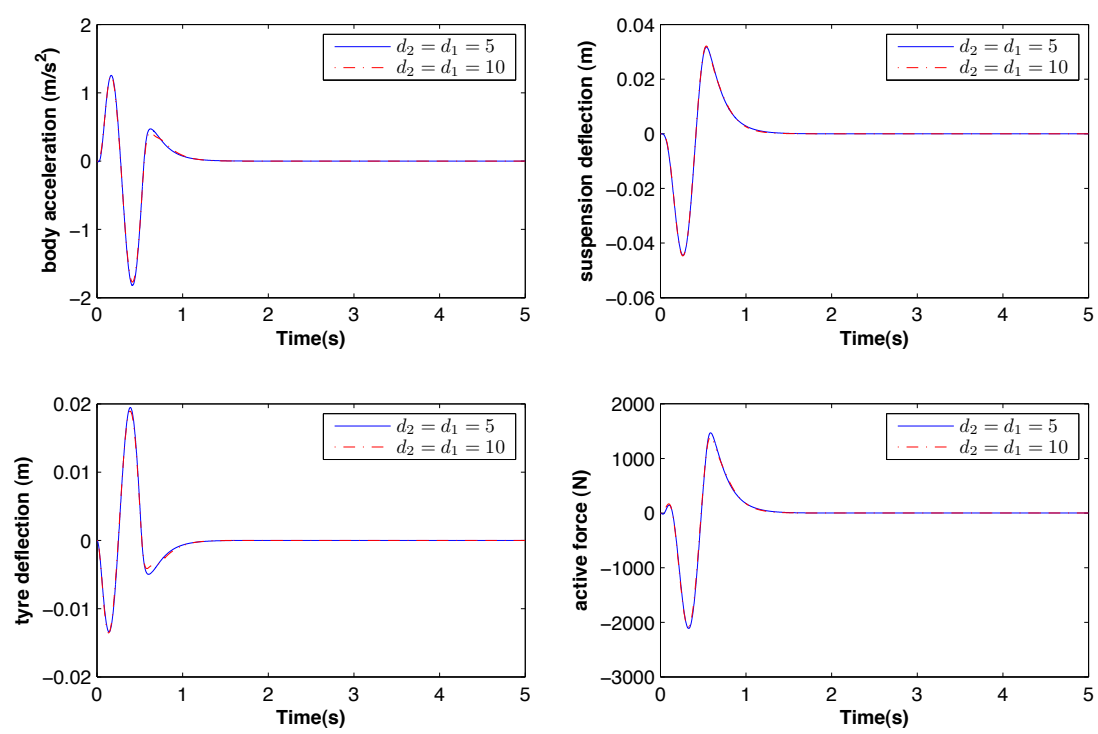

Fig. 3. Vertical accelerations, suspension strokes, tyre deflection and active force for system $(\bar{\delta}=0)$
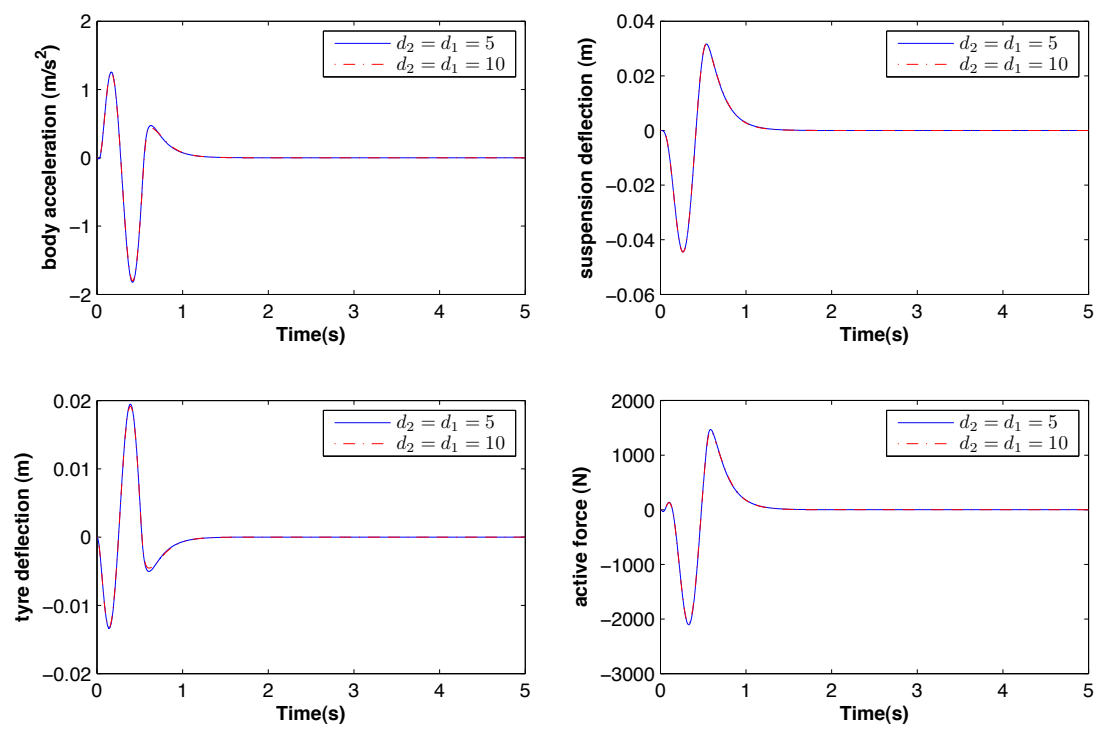

Fig. 4. Vertical accelerations, suspension strokes, tyre deflection and active force for system $(\bar{\delta}=0.5)$ 

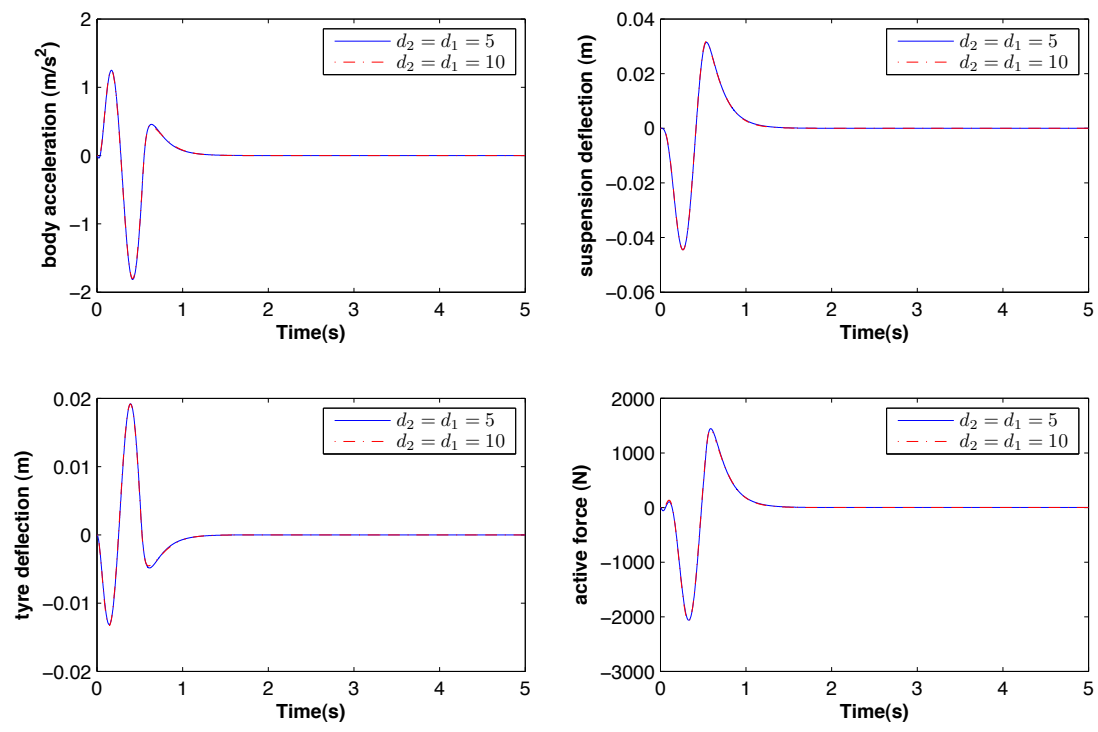

Fig. 5. Vertical accelerations, suspension strokes, tyre deflection and active force for system $(\bar{\delta}=1)$

\section{CONCLUding REMARKS}

This paper has investigated the problem of multi-objective $H_{\infty}$ for active vehicle suspension systems with random actuator delay. The random delay is assumed to be probability distribution and time-varying. The dynamic equations of a quarter-car suspension model have been set up for the control design aim. Based on the stochastic stability theory, we have developed the multi-objective $H_{\infty}$ performance analysis and controller synthesis conditions, which have been cast into a convex optimization problem with LMI constraints via some algebraic manipulations. Then the desired controller has been achieved by solving the corresponding LMIs. Finally, a design example has been given to demonstrate the effectiveness of the proposed controller design approach.

\section{REFERENCES}

[1] H. Gao, J. Lam, and C. Wang, "Multi-objective control of vehicle active suspension systems via load-dependent controllers," Journal of Sound and Vibration, vol. 290, no. 3-5, pp. 654-675, 2006.

[2] H. Gao, W. Sun, and P. Shi, "Robust sampled-data $H_{\infty}$ control for vehicle active suspension systems ," IEEE Transactions on Control Systems Technology, vol. 18, no. 1, pp. 238-245, 2010.

[3] H. Du and N. Zhang, " $H_{\infty}$ control of active vehicle suspensions with actuator time delay," Journal of Sound and Vibration, vol. 301, no. 1-2, pp. 236-252, 2007.

[4] D. Hrovat, "Survey of advanced suspension developments and related optimal control applications," Automatica, vol. 33, no. 10, pp. 1781-1817, 1997. 
[5] A. Alleyne and J. Hedrick, "Nonlinear adaptive control of active suspensions," IEEE Transactions on Control Systems Technology, vol. 3, no. 1, pp. 94-101, 1995.

[6] H. Du, K. Yim Sze, and J. Lam, "Semi-active $H_{\infty}$ control of vehicle suspension with magneto-rheological dampers," Journal of Sound and Vibration, vol. 283, no. 3-5, pp. 981-996, 2005.

[7] M. Yamashita, K. Fujimori, K. Hayakawa, and H. Kimura, "Application of $H_{\infty}$ control to active suspension systems," Automatica, vol. 30, no. 11, pp. 1717-1729, 1994.

[8] N. Al-Holou, T. Lahdhiri, D. Joo, J. Weaver, and F. Al-Abbas, "Sliding mode neural network inference fuzzy logic control foractive suspension systems," IEEE Transactions on Fuzzy Systems, vol. 10, no. 2, pp. 234-246, 2002.

[9] C. Sivrioglu and I. Cansever, "LPV gain-scheduling controller design for a non-linear quarter-vehicle active suspension system," Transactions of the Institute of Measurement and Control, vol. 31, no. 1, pp. 71-95, 2009.

[10] M. ElMadany and Z. Abduljabbar, "Linear quadratic Gaussian control of a quarter-car suspension," Vehicle System Dynamics, vol. 32, no. 6, pp. 479-497, 1999.

[11] I. Fialho and G. Balas, "Road adaptive active suspension design using linear parameter-varying gain-scheduling," IEEE transactions on control systems technology, vol. 10, no. 1, pp. 43-54, 2002.

[12] H. Chen and K. Guo, "Constrained control of active suspensions: an LMI approach," IEEE Transactions on Control Systems Technology, vol. 13, no. 3, pp. 412-421, 2005.

[13] N. Dakev, J. Whidborne, A. Chipperfield, and P. Fleming, "Evolutionary $H_{\infty}$ design of an electromagnetic suspension control system for a maglev vehicle," Proceedings of the Institution of Mechanical Engineers, Part I: Journal of Systems and Control Engineering, vol. 211, no. 5, pp. 345-355, 1997.

[14] H. Chen, Z. Liu, and P. Sun, "Application of constrained $H_{\infty}$ control to active suspension systems on half-car models," Journal of Dynamic Systems, Measurement, and Control, vol. 127, pp. 345-354, 2005.

[15] H. Du, J. Lam, and K. Sze, "Non-fragile output feedback $H_{\infty}$ vehicle suspension control using genetic algorithm," Engineering Applications of Artificial Intelligence, vol. 16, no. 7-8, pp. 667-680, 2003.

[16] K. Gu, V. Kharitonov, and J. Chen, Stability of time-delay systems. Birkhauser, 2003.

[17] A. Agrawal and J. Yang, "Effect of fixed time delay on stability and performance of actively controlled civil engineering structures," Earthquake Engineering \& Structural Dynamics, vol. 26, no. 11, pp. 1169-1185, 1997.

[18] N. Janse van Rensburg, J. Steyn, and P. Els, "Time delay in a semi-active damper: modelling the bypass valve," Journal of Terramechanics, vol. 39, no. 1, pp. 35-45, 2002.

[19] A. Vahidi and A. Eskandarian, "Predictive time-delay control of active suspensions," Journal of Vibration and Control, vol. 7, pp. 1195-1211, 2001.

[20] N. Jalili and E. Esmailzadeh, "Optimum active vehicle suspensions with actuator time delay," Trans. ASME, J. Dynamic Systems, Measmt, Control, vol. 123, no. 1, pp. 54-61, 2001.

[21] H. Du and N. Zhang, "Constrained $H_{\infty}$ control of active suspension for a half-car model with a time delay in control," Proceedings of the Institution of Mechanical Engineers, Part D: Journal of Automobile Engineering, vol. 222, no. 5, pp. 665-684, 2008.

[22] H. Du, N. Zhang, and J. Lam, "Parameter-dependent input-delayed control of uncertain vehicle suspensions," Journal of Sound and Vibration, vol. 317, no. 3-5, pp. 537-556, 2008.

[23] I. Molina-Cristobal, A. Griffin, P. Fleming, and D. Owens, "Linear matrix inequalities and evolutionary optimization in multiobjective control," International Journal of Systems Science, vol. 37, pp. 513-522, 2006. 
[24] F. Yang, Z. Wang, Y. Hung, and M. Gani, " $H_{\infty}$ control for networked systems with random communication delays," IEEE Transactions on Automatic Control, vol. 51, no. 3, pp. 511-518, 2006.

[25] D. Yue, E. Tian, Y. Zhang, and C. Peng, "Delay-distribution-dependent stability and stabilization of T-S fuzzy systems with probabilistic interval delay," IEEE Transactions on Systems, Man, and Cybernetics, Part B: Cybernetics, vol. 39, no. 2, pp. 503-516, 2009.

[26] D. Yue, E. Tian, Z. Wang, and J. Lam, "Stabilization of systems with probabilistic interval input delays and its applications to networked control systems ," IEEE Transactions on Systems, Man, and Cybernetics, Part A: Systems and Humans, vol. 39, no. 4, pp. 939-945, 2009.

[27] C. Lin, Z. Wang, and F. Yang, "Observer-based networked control for continuous-time systems with random sensor delays," Automatica, vol. 45, no. 2, pp. 578-584, 2009.

[28] X. Mao, "Stability of stochastic differential equations with Markovian switching," Stochastic Processes and Their Applications, vol. 79, no. 1, pp. 45-67, 1999. 